\title{
Control Strategies for Soft Robotic Manipulators: A Survey
}

\author{
Thomas George Thuruthel, Yasmin Ansari, Egidio Falotico, and Cecilia Laschi
}

\begin{abstract}
With the rise of soft robotics technology and applications, there have been increasing interests in the development of controllers appropriate for their particular design. Being fundamentally different from traditional rigid robots, there is still not a unified framework for the design, analysis, and control of these high-dimensional robots. This review article attempts to provide an insight into various controllers developed for continuum/soft robots as a guideline for future applications in the soft robotics field. A comprehensive assessment of various control strategies and an insight into the future areas of research in this field are presented.
\end{abstract}

Keywords: continuum robots, soft robots, manipulation, dynamic controllers, kinematic controllers, machine learning

\section{Introduction}

B IOLOGICAL ORGANISMS EXPLOIT SOFTNESS of the body for compliance to reduce the complexity in interacting with the environment. This characteristic is promising to advance robotic systems to operate robustly and adaptively in unstructured environments. Incorporating softness in robotic systems, in particular robotic manipulators, the focus of this article, is studied under the domain of "soft robotics." This term is associated with two distinct design approaches: (1) compliant joints (active or passive) within rigid-link robots ${ }^{1,2}$ and (2) continuum robotic manipulators. ${ }^{3}$ The discussion in this article is restricted to the latter one.

Although the field of continuum robotic manipulators was founded in the 1960s, a formal research on the design and control can be dated back to the early 1990s. These systems are the result of the evolution of manipulator design from discrete mechanisms constructed from a series of rigid links to mechanisms without rigid links but rather with elastic structures capable of continuous bending along their length depicted in Figure 1.

A novel subdomain of continuum manipulators, referred to as "soft robotic manipulators,", 4,5 has been rapidly growing in the past decade since roboticists found inspiration in boneless biological organisms such as octopus arms, which are able to exploit the "mechanically intelligent" arrangement of just their muscles to exhibit dexterous advanced manipulation capabilities in cluttered environments. This has been translated into new range of continuum manipulators made up of soft materials such as silicone due to their ability to undergo a large deformation under normal operation. The underlying idea is to use principles of embodied intelligence ${ }^{6}$ and morphological computation ${ }^{7}$ to exploit the soft material properties to enable machines with properties such as inherent compliance, variable stiffness, and highly dexterous motion in an unstructured environment. The resulting systems have the ability to simplify a wide range of well-known complex tasks. In addition, they offer a low-cost alternative to numerous robotic applications. ${ }^{5}$ Furthermore, the deformability of the soft material offers compliance, which facilitates safe human-robot interaction in comparison to the rigid counterparts. These desirable characteristics are the fundamental reason behind the rapidly increasing demand in industrial, surgical, and assistive applications.

However, the long-term success for the practical application of these systems is dependent on the development of real-time kinematic and/or dynamic controllers that facilitate fast, reliable, accurate, and energy-efficient control. This is nontrivial because (1) unlike rigid manipulators, the movement of which can be specified by three translations and three rotations, elastic deformation of soft robotic manipulators results in virtually infinite degrees-of-freedom (DoF) motions, (bending, extension, contraction, torsion, buckling, etc.); (2) the material properties exhibit nonlinear characteristics such as compliance and hysteresis that restrict high-frequency control;

Soft Robotics Laboratory, The Biorobotics Institute, Scuola Superiore Sant'Anna, Pisa, Italy. 


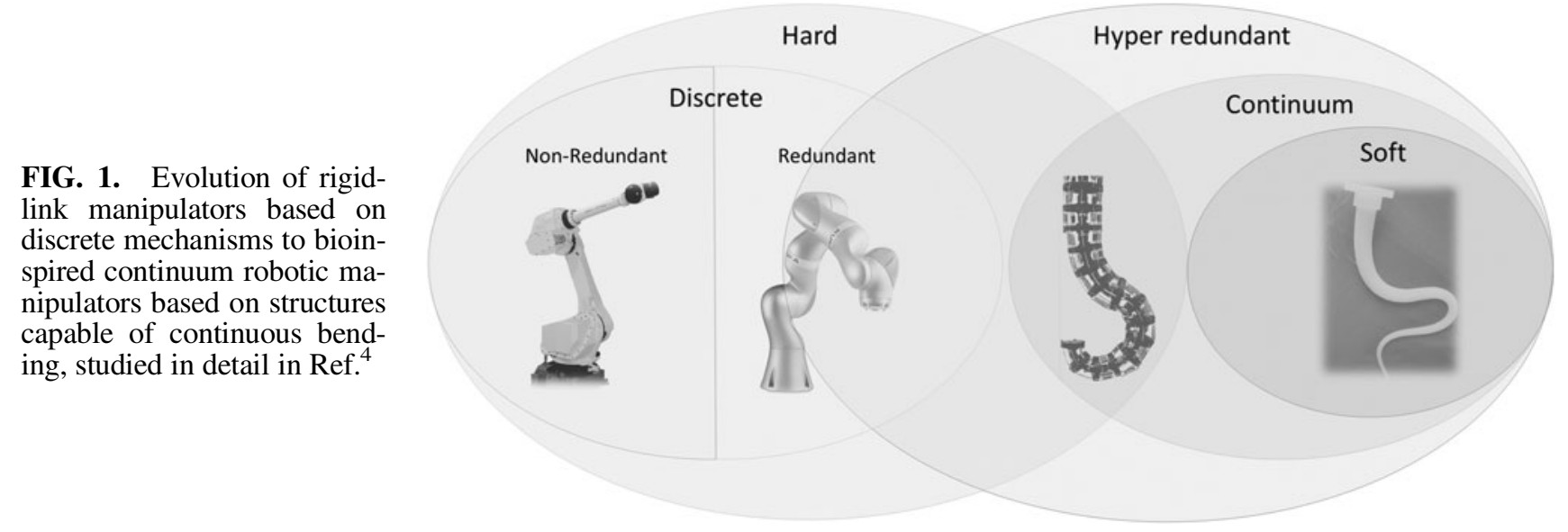

(3) the wide range of design and actuation techniques, which makes each of these robots have unique properties (refer to Ref. $^{8}$ for a detailed review on design and actuation technologies for soft robots). However, as this is an active field of research still in its infancy, the fundamental purpose of this survey is to provide an in-depth assessment of various control strategies established within the domain of continuum robotic manipulators, in the past decade, with the aim to segue into a guide for researchers toward possible directions for developing controllers for soft robotic manipulators.

Comprehensive reviews of control strategies for continuum robotic manipulators ${ }^{3,9-12}$ are primarily focused on design, fabrication, modeling, and sensing. However, there is no in-depth analysis of the control approaches that have emerged over the years. Furthermore, they lack focus on the recent development of controllers via model-free approaches. This survey tries to first enumerate all such developments in this field in the past decade; second, it aims to provide a unified overview of key terminologies, advantages, and drawbacks of these controllers; and finally, we aim to summarize these concepts in a table that, in addition to a systematic review, also provides the readers an overview into the chronological developments that have led to the current landscape and prospects of development in this domain. As the focus of this article is restricted to controllers developed within the domain of continuum manipulators that can be adopted for soft robotic manipulators, the article does not dwell much into modeling techniques, theoretical studies, wearable robots, and concentric tube robots. The role of sensing and variable stiffness actuation for control is also beyond the scope of this article.

\section{Preliminaries}

Although a lot of classic terminologies used for rigid robots can be directly adapted to this field, special care must be given to understand the applicability and limitations of these terms. Consequently, we first state key terminologies and their corresponding definitions that will be used throughout the article to describe the controllers in a unified manner. Next, we lay out the classification schema used to systemically analyze the controllers summarized in Table 1.

\section{Definitions}

Figure 2 provides the definitions and terminologies that we will be referring to throughout the article. The purpose of the figure is to give the readers an idea of the different levels of mapping involved in the control of a continuum/soft manipulator and its differences from traditional rigid robot control.

\section{Classification schema}

1. Modeling approach:

a. Model-based controllers rely on analytical models for deriving the controller.

b. Model-free controllers use machine learning techniques or empirical methods.

c. Hybrid controllers combine model-based and modelfree approaches.

2. Design:

a. Actuation: tendon-driven, pneumatic, interleaved, simulated platforms.

b. Actuation details: number of segments/actuators, arrangement, shape, material.

c. Manipulator details: intended applications.

3. Control:

a. Operating space:

i. Low-level: joint and/or actuator space.

ii. Mid-level: inverse static/kinematic, ${ }^{*}$ dynamic. $^{\dagger}$ iii. High-level: path planning.

b. Controller details: tested for planar/nonplanar applications, required sensors.

c. Performance: error measurements, theoretical error convergence, stability.

\section{Model-Based Static Controllers}

Soft robots present a formidable challenge to modeling due to their high dimensionality. Nonetheless, tractable kinematic models can be developed by adopting a steady-state assumption; that is, under force equilibrium, the full configuration

* Static controllers are time invariant controllers where the control variables are zero order.

${ }^{\dagger}$ Dynamic controllers consider the configuration space and/or task space variable velocities in the control algorithm.

${ }^{7}$ High-level controllers that prescribe the reference path in task space are primarily application based. Since the area of soft robotics is still in its incipient phase, a review on high-level controllers is beyond the scope of this article. 


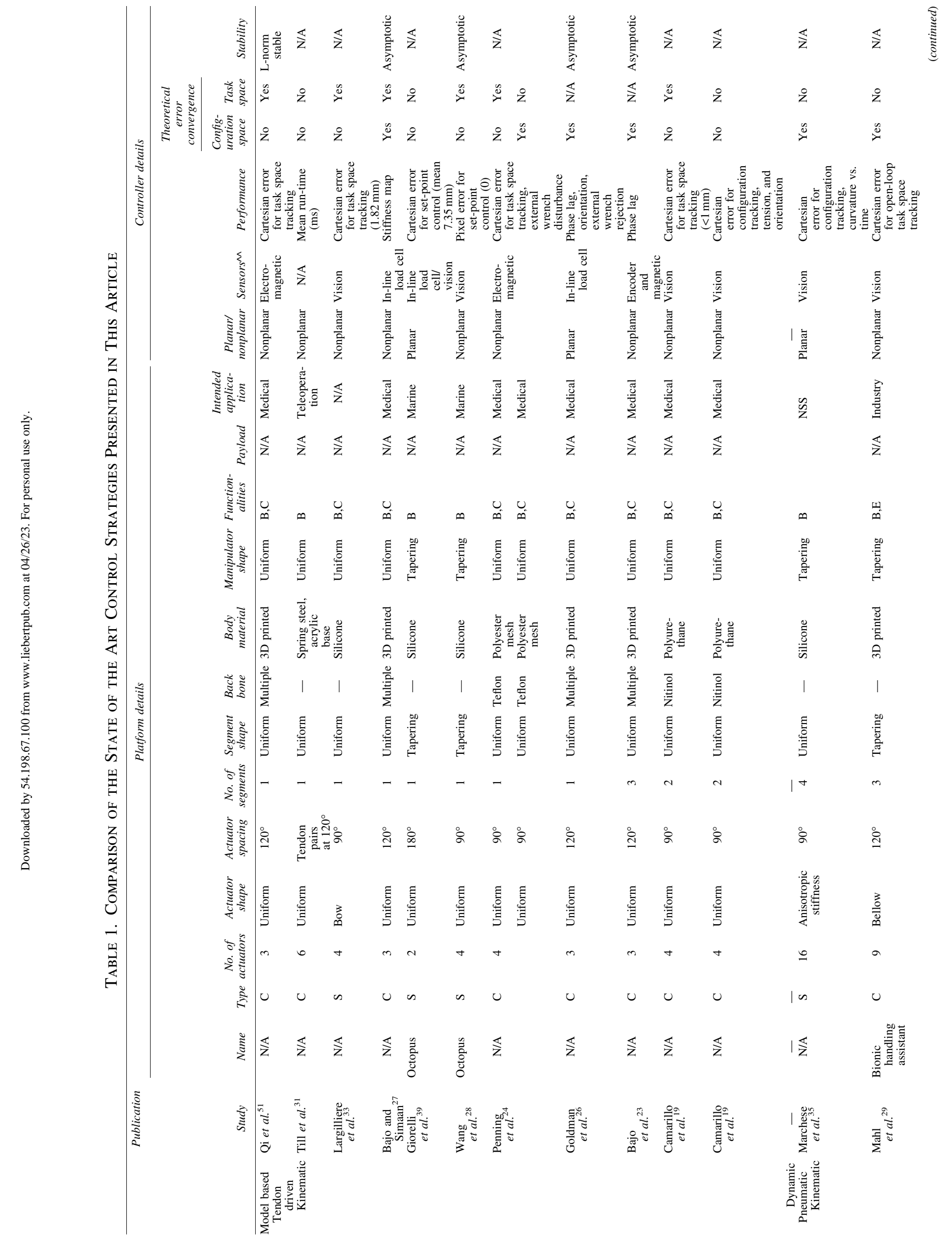




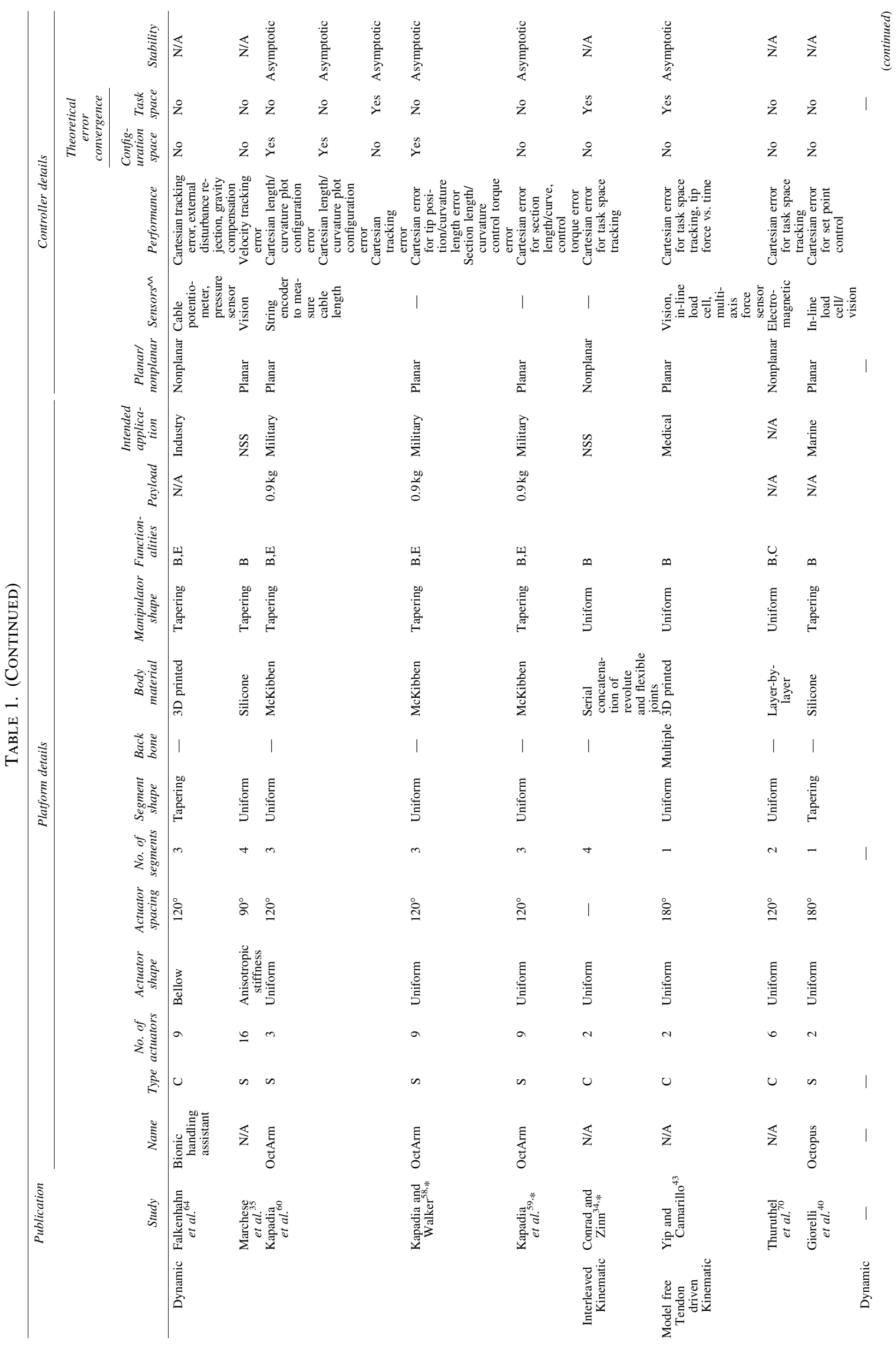




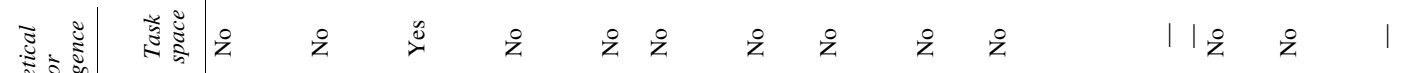

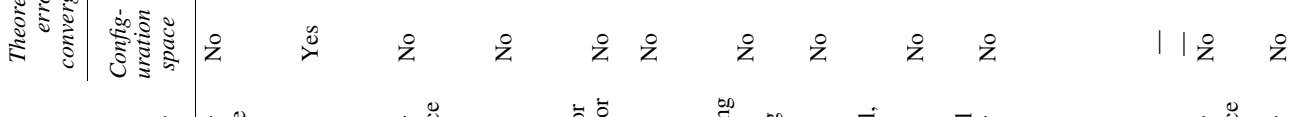

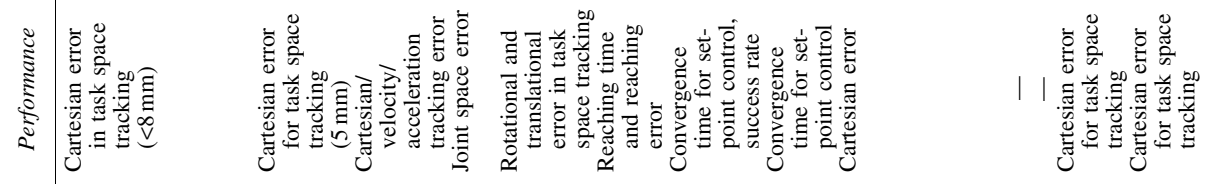

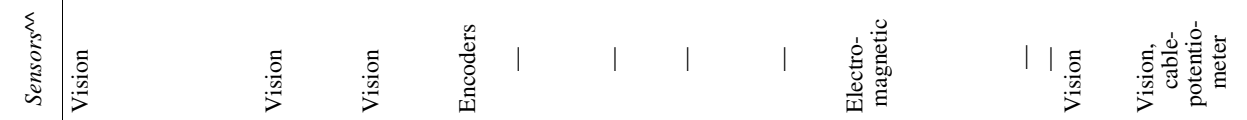

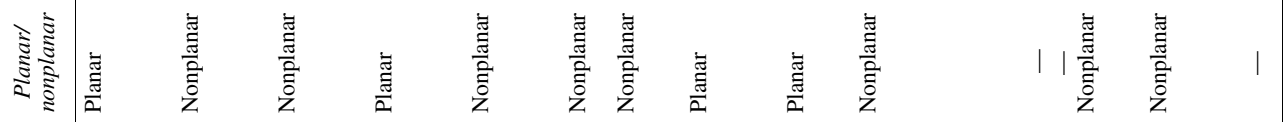

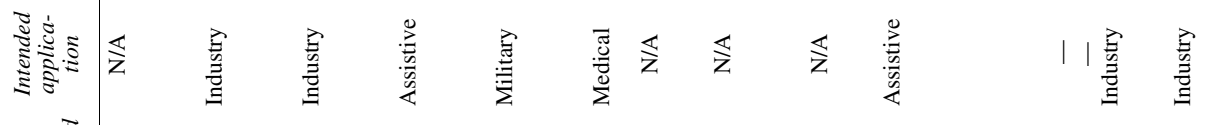

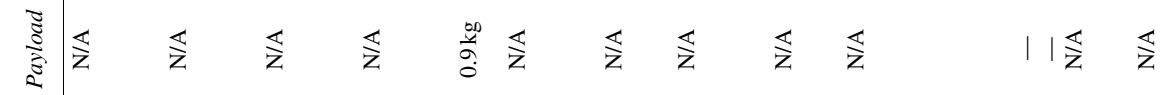

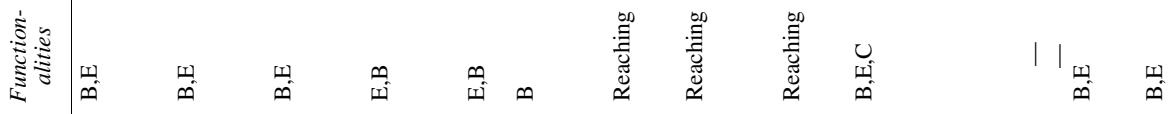

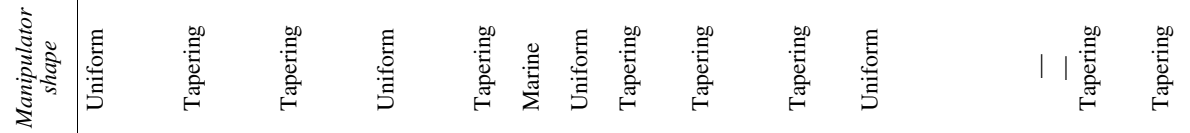

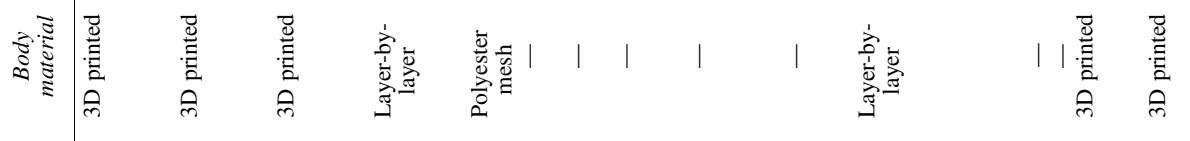

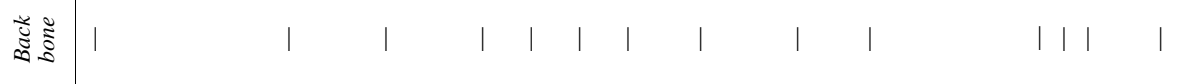

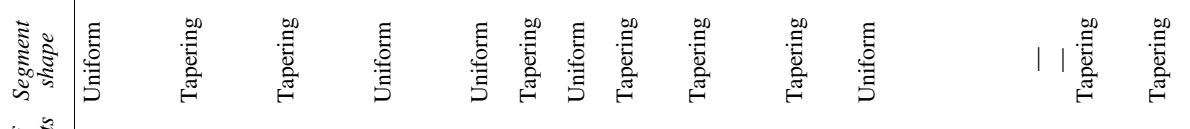

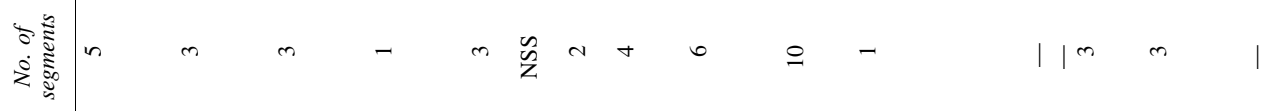

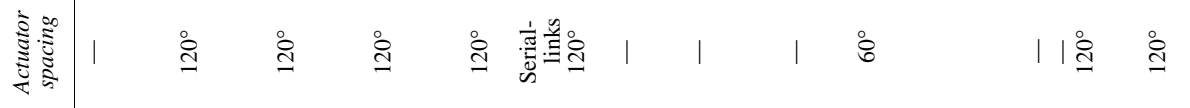

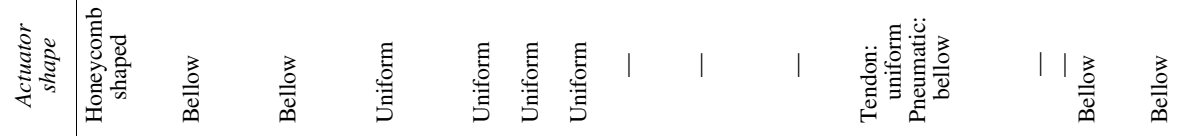

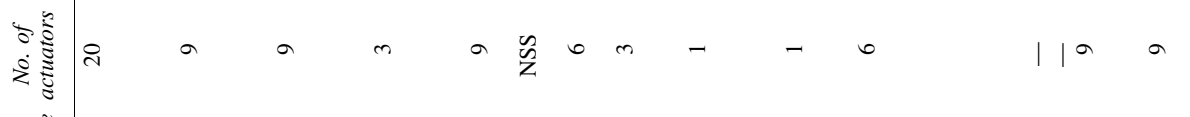

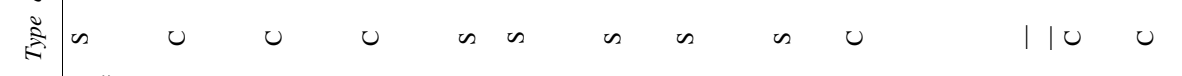

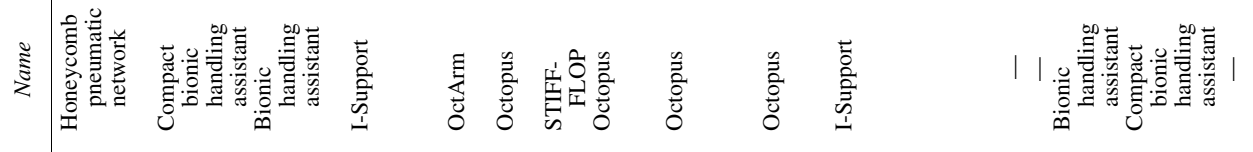

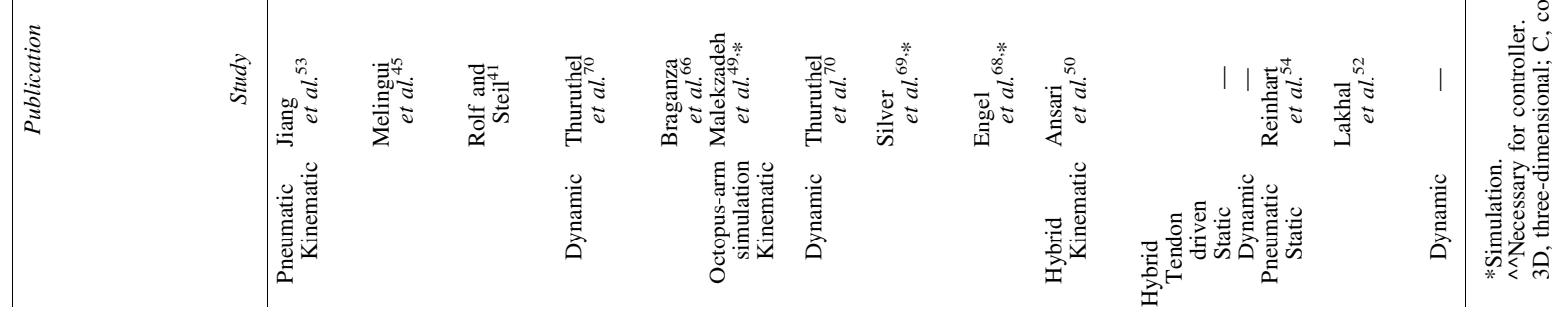




\begin{tabular}{|c|c|c|c|}
\hline Operating Space & Definition & Pneumatic & Tendon-Driven \\
\hline Actuator Space & $\boldsymbol{q} \in \mathbb{R}^{k}$ & $\begin{array}{c}q \propto E g: \text { chamber pressure or volume or both } \\
k=\text { number of actuators }\end{array}$ & $\begin{array}{c}q \propto \text { Eg: motor position/torques } \\
k=\text { number of actuators }\end{array}$ \\
\hline Joint Space & $\boldsymbol{\varsigma} \in \mathbb{R}^{l}$ & $\begin{array}{c}\varsigma \propto E g: \text { cable }- \text { potentiometers } / \text { tension } \\
\quad l \geq \text { number of actuators }\end{array}$ & $\begin{array}{c}\varsigma \propto E g: \text { cable length/tension } \\
l \geq \text { number of actuators }\end{array}$ \\
\hline Configuration Space & $\boldsymbol{\zeta} \in \mathbb{R}^{m}$ & \multicolumn{2}{|c|}{$\begin{array}{l}\zeta \propto \text { no. of independent physical parameters that define the configuration of the manipulator } \\
\qquad m=\text { l under steady-state conditions * }\end{array}$} \\
\hline Task Space & $\boldsymbol{x} \in \mathbb{R}^{n}$ & \multicolumn{2}{|c|}{$\begin{array}{c}x \propto \text { position or pose or forces applied at end }- \text { effector } \\
n=\text { dimension of target variable }\end{array}$} \\
\hline
\end{tabular}

*dimensions of uniform and nonuniform manipulators remain the same even under gravitational loading, albeit represented differently Note: A manipulator is considered redundant when $n \leq l$

Note: In Fig 4 -9, the operating space has been abbreviated using the first letter of each word. So for eg. Actuator Space is mentioned as A.S.

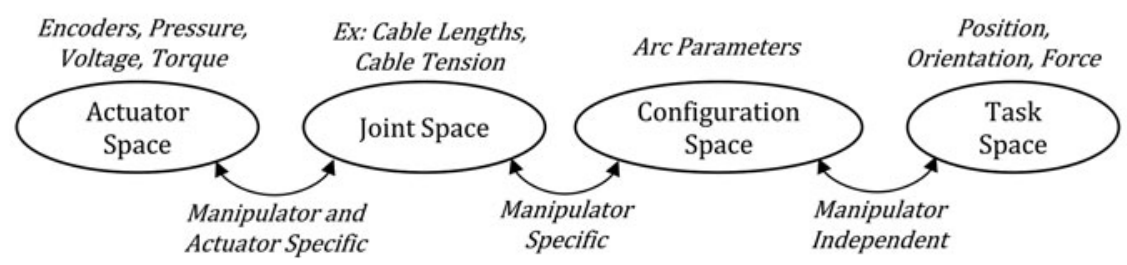

FIG. 2. Operating spaces of a continuum manipulator and their definitions.

of the soft manipulator can be defined by a low-dimensional state space representation. In all the articles that are reviewed, this assumption is valid and therefore we interchangeably use the term "statics" and "kinematics" even though this is not a common practice in traditional robotics.

The simplest and most commonly used kinematic/steadystate model assumes that the configuration space of a threedimensional (3D) continuum/soft module can be parameterized by three variables, more commonly referred to as the constant curvature (CC) approximation. ${ }^{13}$ It reduces an infinite dimensional structure into just 3D, thereby ignoring a large portion of the manipulator dynamics. This has been found to be a very good approximation if (1) the manipulator is uniform in shape and symmetric in actuation design, (2) external loading effects are negligible, and (3) torsional effects are minimal. It is important to realize that the $\mathrm{CC}$ model arises due to a constant strain approximation along the length of the manipulator and therefore is a model truly valid only in the steady-state condition. ${ }^{14}$ In Ref., ${ }^{15}$ it was demonstrated that the variations in the kinematic manipulability ellipsoid are very less when going from a low-dimension to a highdimension representation of the manipulator configuration. This could explain the relative success of the CC model. For multisection continuum/soft manipulators, each $\mathrm{CC}$ section can be stitched together to provide the piecewise constant curvature (PCC) model. ${ }^{16}$ Concurrently, a more complex modeling approach using beam theory was pursued using beam theory ${ }^{14}$ and Cosserat rod theory. ${ }^{17}$ However, the improvement in accuracy attained by a more complex model was not significant enough considering their computational and sensing cost and therefore have been limited in their usage.

Once a kinematic model is established, it is necessary to invert the kinematics to obtain the desired actuator or configuration space variables. This can be pretty straightforward and has been widely studied for rigid manipulators and can be done with differential inverse kinematics (IK), ${ }^{16,18}$ by direct inversion, ${ }^{19}$ or by optimization. ${ }^{20}$ Furthermore, a lowlevel controller takes care of tracking in the actuator/joint space, usually using a simple linear closed loop controller. In addition, actuator compensation techniques might have to be used because of the presence of friction, hysteresis, ${ }^{21}$ or tendon coupling ${ }^{22}$ that can cause deviations from the forward steady-state model.

The need to model and compensate for slackening tendon load coupling and tendon path coupling for multisection manipulators was first addressed in Ref. ${ }^{19}$ A numerically estimated static model used for the forward model and inverse model was obtained by optimization. However, there still lacked an expression for friction effects and the approach was used only for configuration tracking.

One of the fundamental modeling difficulties involved with cable-driven actuators is the path coupling among sections. For independent actuation methods, only the load coupling needs to be considered. Further on, researchers started investigating the importance of sensors for compensating modeling uncertainties without the necessity for formulating very complex compensation techniques. ${ }^{20,23}$ As an extension of Ref., ${ }^{19}$ a closed-loop task space controller was proposed and 


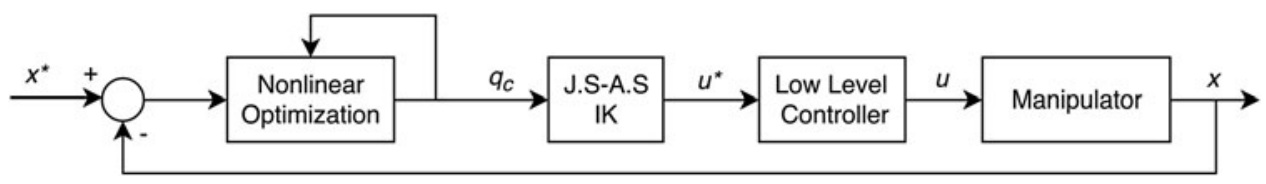

FIG. 3. A closed-loop task space controller implementation. An asterisk $(*)$ represents the desired variable value, and a subscript " $c$ " represents the commanded variable value. IK, inverse kinematics.

experimentally validated for the first time in Ref. ${ }^{20}$ with a 5DoF per section kinematic model. For this, the IK problem is formulated as a constrained nonlinear optimization problem where the desired joint configuration that reduces the current tracking error is estimated while satisfying the forward kinematic model and cable tension constraints (to avoid slacking). By representing the kinematics in the velocity level, their approach gains leverage in terms of higher accuracy (submillimeter) and robustness to model uncertainties, but would need to solve a high-level path planner (Fig. 3). The downside of the direct task space controller is instability (can be solved by lower control frequency; $5 \mathrm{~Hz}$ for Ref. ${ }^{20}$ ) and slower convergence.

In Ref., ${ }^{23}$ a configuration space controller is proposed, which uses external sensory information about the configuration and internal sensory information about the joint variables to achieve asymptotic tracking of a stationary configuration target. By providing additional tracking information and framing a cascaded controller, they were able to reduce coupling effects and decrease the phase lag while tracking a time varying trajectory. Being a configuration space feedback controller, the control loop was run faster at $150 \mathrm{~Hz}$. Interestingly, significant phase lag was observed even for tasks at $2 \mathrm{~Hz}$ and this is highly undesirable at the low level. Similarly in Ref., ${ }^{24}$ two closedloop controllers in the task space (Fig. 4) and joint space (Fig. 5) were compared. The advantage of a direct closed-loop task space controller is that it can provide asymptotic convergence of the error even with model uncertainties. On the contrary, a joint space controller can offer independent control of the joint variables allowing for individual tuning and hence more stability, especially if the joint/actuator motions are discrete. Note that for all the abovementioned controllers, there is also a closed-loop actuator space controller, usually a servo controller, which is assumed to provide perfect tracking. All these methods rely on the $\mathrm{CC}$ approximation for modeling.

Following the strong coupling between continuum manipulator's kinematic and static force model, controllers foraying into compliance/force control started to emerge. ${ }^{25-27}$ In Ref., ${ }^{26}$ it was demonstrated that by knowing the current internal actuation forces and the configuration space variables, an estimate of the external generalized forces can be formed. Using the estimate of the external force and the compliance matrix (maps the change in actuator forces to the tip wrenches), a configuration space controller for reducing tip forces for surgical purposes was proposed. As an extension of Ref., ${ }^{26}$ a hybrid position/force controller in the configuration space was realized in Ref. ${ }^{27}$ (Fig. 6). Desired twist and wrench vectors are projected orthogonally (for decoupling the control effort into feasible motions) and transformed to configuration space references using differential IK and the configuration space compliance matrix (maps the change in configuration space variables to the tip wrenches), respectively.

Hybrid position/force control was realized in Ref. ${ }^{25}$ without the need of force sensors. This was done by numerically calculating the transformation matrix that maps the transformation from the tip of an unloaded continuum manipulator to the tip position when acted on by external forces using Cosserat rod theory. With the transformation formulation, the desired joint position that attains a particular end effector force and orientation was estimated using fixed point iteration. Compensating model deviations due to friction and other nonlinear material behavior remains an open research topic.

Further on, researchers started to focus on more complex kinematic formulations by extending the CC model, mostly due to the rise of biologically similar tapering continuum robots. The first such method was the use of the variable constant curvature (VCC) approximation, which models a single module as $n$ segments of $\mathrm{CC}$, where the curvature of each segment depends on the radius of the segment, thus creating a high-dimensional configuration space. ${ }^{28-30}$ The VCC model for a three-section, pneumatically actuated continuum robot, with the procedure for segmentation of the sections, was first elucidated in Refs. ${ }^{29,30}$ A resolved motion rate algorithm was used for the closed-loop control of the robot due to the double advantage of redundancy resolution and the robustness it provides to model uncertainties (Fig. 7).

Visual servo control of a two-dimensional (2D) image feature point in 3D space using a cable-driven soft conical manipulator was proposed using the VCC model in Ref. ${ }^{28} \mathrm{~A}$ differential kinematic-based controller, similar to the one in Ref., ${ }^{29}$ with the control objective of reducing the feature point tracking error was proposed. An adaptive algorithm for depth estimation was also described. Similarly, efficient numerical techniques for solving in real time the complex Cosserat models were detailed in Ref., ${ }^{31}$ however, no control experiments were demonstrated.

Contrary to ongoing developments, the use of simplified kinematic models for control was proposed in Ref. ${ }^{32}$ The idea behind this is that the reduced accuracy due to the inaccurate kinematics can be compensated or even improved with the increased control cycle frequency gained due to the low

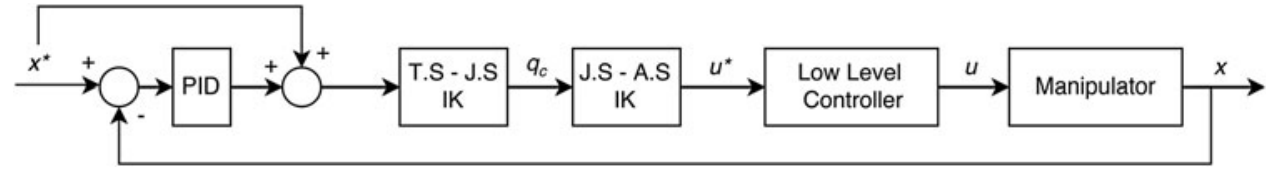

FIG. 4. A closed-loop task space controller implementation. PID, proportional integral derivative. 
FIG. 5. A task space controller implemented by closedloop control in the joint space. A subscript " $c$ " represents the variable estimate.

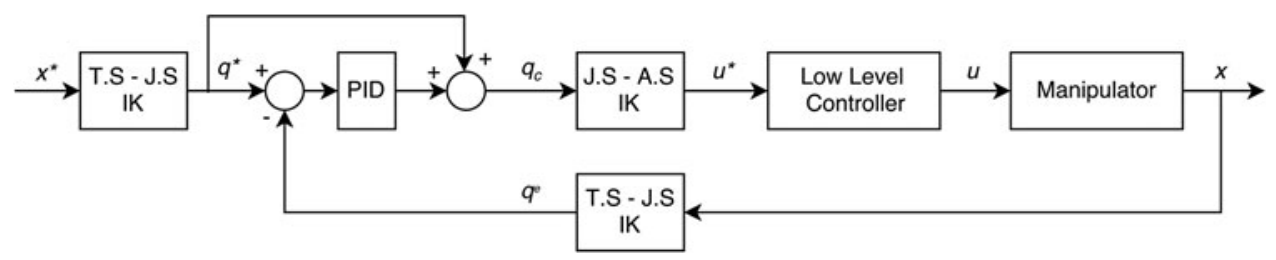

computational cost. However, the method was validated only on simulations and would not be directly transferable to a real setup at the same frequency without considering the low-level dynamics as observed in Ref. ${ }^{23}$ On the other spectrum, a numerically exact approach for statics modeling using asynchronous finite element analysis (FEM) was described in Ref. ${ }^{33}$ Optimization using quadric programming (QP) algorithm was used to obtain the inverse solution, which is used to control the actuators at high frequencies while a low-frequency loop FEM simulation feeds the inputs to the QP solver.

Recent developments in terms of model-based static controllers are factored on the design aspects. A closed-loop task space controller was applied on an interleaved continuum-rigid manipulator in Ref. ${ }^{34}$ The main idea of the approach is to use the well-behaved rigid links in tandem with the flexible elements to compensate for the errors obtained while tracking a desired tip position thereby obtaining much lower bound on the tracking error. However, the scalability of such designs for high-dimensional systems is still a question mark. Currently, the manipulator is designed with the rigid components set up at the base, but it will be tricky to add further components in serial.

On the contrary, kinematic control of a pneumatically actuated soft manipulator entirely made from a low durometer elastomer was detailed in Ref. ${ }^{35}$ The control architecture is similar to Ref. ${ }^{23}$ and tries to achieve tracking of configuration space variables using a cascaded proportional integralproportional integral derivative in the configuration space and actuator space (cylinder displacement, in this case), respectively. The task space to configuration space IK obtained a nonlinear constrained optimization. Both the abovementioned approaches used the $\mathrm{CC}$ approximation for the configuration space model.

\section{Summary of model-based static controllers}

Model-based static controllers are currently the most widely used and studied strategy for control of continuum/ soft robots. Majority of the model-based controllers rely on the CC approximation since more complex models are computationally expensive and are design specific. However, with validation of the $\mathrm{CC}$ model for a completely soft robot ${ }^{35}$ and its wide application for control of many continuum/soft robots, it is still one of the most reliable and easily applicable methods for static control of uniform, low-mass manipulators. More complex methodologies have not achieved exceptional performance improvements because of their computational cost and numerous parameters that have to be estimated. This was also observed in recent comparisons among various modeling approaches on the same platform. ${ }^{36}$ In light of this, model-free approaches provide an alternative means to develop more complex yet accurate, design-specific models without any prior knowledge about the underlying structure.

In terms of operating space, a closed-loop configuration space controller or joint space controller would provide more stable and faster controllers, however, cannot guarantee error convergence (unless there is a perfect forward model available). Closed-loop task space controllers can theoretically provide the best accuracy. In terms of actuation, tendondriven systems are more difficult to model, whereas pneumatic manipulators would need more sensors.

\section{Model-Free Static Controllers}

Model-free-based approaches for control of continuum/ soft robots are a relatively new field and offer a wide range of possibilities. Although these data-dependent methods have been used effectively in the field of rigid manipulators, ${ }^{37}$ the same cannot be said for continuum manipulators even though model-free approaches intuitively should fare better in this case.

The first usage of a model-free approach for development of a static controller was proposed in Ref. ${ }^{38}$ The approach was a straightforward direct learning of the inverse statics of a nonredundant (with respect to the actuator space and task space) soft robot using a neural network. Although the method was correctly able to predict the reference cable tensions for reaching a target in the task space in simulations, the approach cannot be scaled for redundant systems and does not consider the stochastic nature of real soft robots. An experimental validation of the same approach was done in Ref. ${ }^{39}$ for a $2-\mathrm{DoF}$ and a $3-\mathrm{DoF}^{40}$ cable-driven soft manipulator and compared with an IK model derived from a numerically exact model.
FIG. 6. Closed-loop task space control of position and force implementation. A subscript " $v$ " represents the firstorder derivative of the variable.

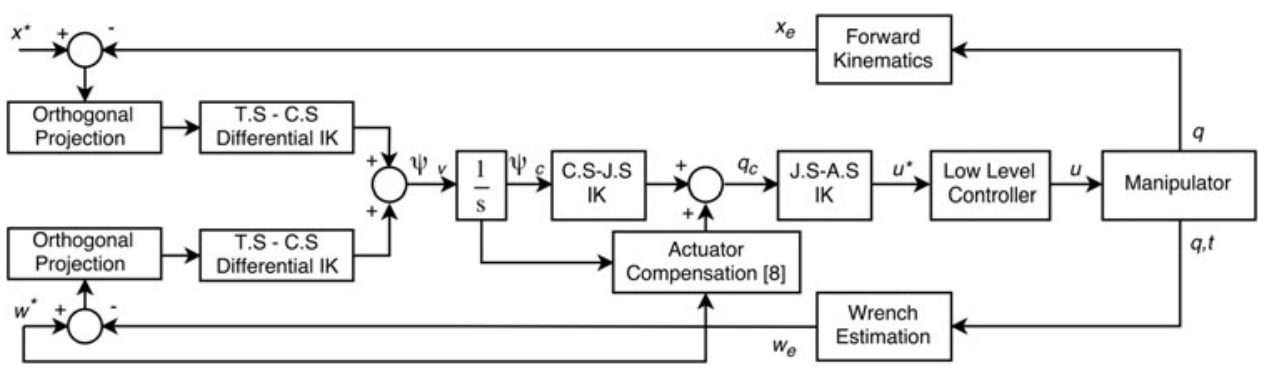




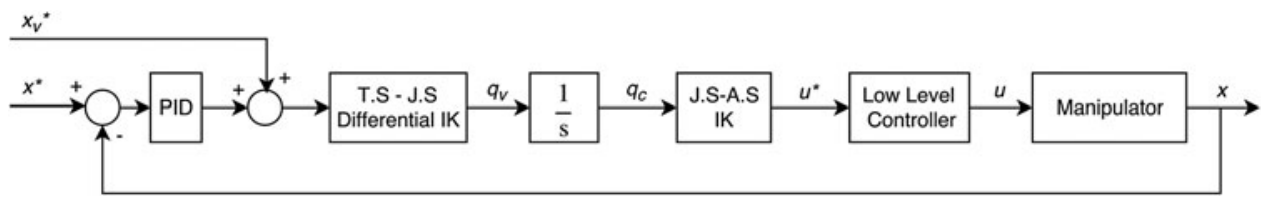

FIG. 7. First-order resolved motion rate algorithm for closed-loop task space control. Note the similarity to the first implementation in Figure 3. The additional feedforward component allows for faster convergence.

Interestingly, the simple neural network-based approach performed significantly better than the computational complex analytical method. The final controller is similar to the diagram shown in Figure 8 without the feedback component.

An efficient exploration algorithm for generating samples for IK learning was proposed in Ref. ${ }^{41}$ The main idea is to use goal babbling to generate samples from the task space to actuator space for high-dimensional redundant systems. Since the exploration is goal oriented, it can allow for efficient exploration (by avoiding revisiting an explored task space/actuator space region) and in selecting a desired redundancy resolution scheme. Finally, self-organizing maps are used to learn the IK mapping with generated samples. A feedback scheme for reducing tracking error due to the stochasticity of model is implemented by virtually shifting the target positions proportional to the error in tracking to generate modified reference positions (Fig. 8).

A highly robust, accurate, and generic approach for closedloop task space control of continuum robots was proposed in Ref. $^{42}$ (Fig. 9). The article proposes an optimal control strategy based on empirical estimation of the kinematic Jacobian matrix online by incrementally moving each actuator. Optimization is done to minimize the control effort and to keep the cables taut. There is no internal model used for control, and therefore, the authors have called the approach as a "model-less" technique. Although such a strategy solves a lot of difficulties in the control of continuum robots, even allowing manipulation in an unstructured environment, the very low control frequency is of practical concern. The same principle was extended for hybrid force/position control in Ref., ${ }^{43}$ where the stiffness matrix is also computed empirically. Similar to other hybrid force/position controllers, the reference position and forces are projected orthogonally when the manipulator is in contact.

Recent model-free approaches have mostly focused on learning the IK representation of continuum robots. In Ref., ${ }^{44}$ an approach for learning the direct mapping between task space and joint space (potentiometer voltage, in this case) is proposed. This involves learning the forward kinematic model first using a neural network and then inverting this learned network using distal supervised learning. However, this approach did not consider the stochasticity of the manipulator and did not implement a feedback error correction scheme. As an improvement of the previous work, in Ref., ${ }^{45}$ the authors try to address the stochasticity of the mapping between the joint space (potentiometer values) and actuator space (chamber pressures) by developing an adaptive subcontroller. This is because for the case of tendon-driven actuation, the actuator space and joint space are linearly related, whereas for pneumatic actuation, an additional nonlinear mapping between the actuator space and the joint space must also be considered. The subcontroller comprises a modified Elman neural network, which emulates the actuator kinematics, and a multilayer perceptron controller that learns to control the actuator variables accordingly. However, the kinematic mapping between the joint space and task space is considered to be nonstochastic, which is not necessarily the case.

Another technique for learning the IK was proposed in Refs., ${ }^{46,47}$ where the IK problem is formulated like a differential IK problem using local mappings. This allowed for redundancy resolution as well as reducing stochastic effects. However, the approach was validated only by simulations on a continuum ${ }^{46}$ and soft arm. ${ }^{47}$ Another advantage of such an approach is that it allows multiple solutions to the IK problem globally and can work even if some of the actuators are nonfunctional after the learning process. A similar modeling method strengthened with a feedback controller was experimentally validated in Ref. ${ }^{48}$ It was also observed that even with a simple feedback controller, intelligent behaviors can be obtained in an unstructured environment.

An attempt toward transfer learning has also been made, however, limited to simulation. ${ }^{49}$ Authors develop an algorithm to transfer the reaching skills from a simulated non-CC octopus arm to a simulated CC soft robotic manipulator. The idea is to design dynamic motion primitives through a weighted combination of Gaussian functions representing the joint distribution of the data. This is combined with a statistical regression approach making it robust to external perturbations in the environment. Although this approach seems promising, it requires more experimental work to demonstrate its potential.

In a recent work, ${ }^{50}$ the authors optimize multiple objectives within a reinforcement learning architecture to learn deterministic stationary policies for a soft robot arm module. Although it works in high dimensions, it is sensitive to external disturbances. An attempt toward fuzzy logic-based controllers was attempted in Ref. ${ }^{51}$ The idea was to develop numerical estimates of the kinematic Jacobian using prior knowledge-based local approximations and interpolation functions. This allows for faster computation, but the advantage of such a method over data-driven machine learning approaches is not apparent. Finally, a hybrid controller

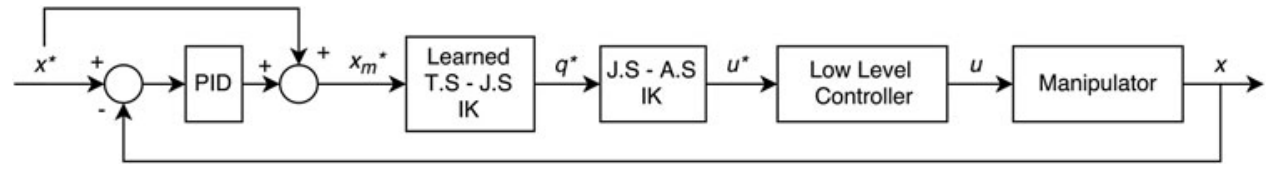

FIG. 8. A general modelfree closed-loop task space controller implementation. A subscript " $m$ " represents an auxiliary variable. 


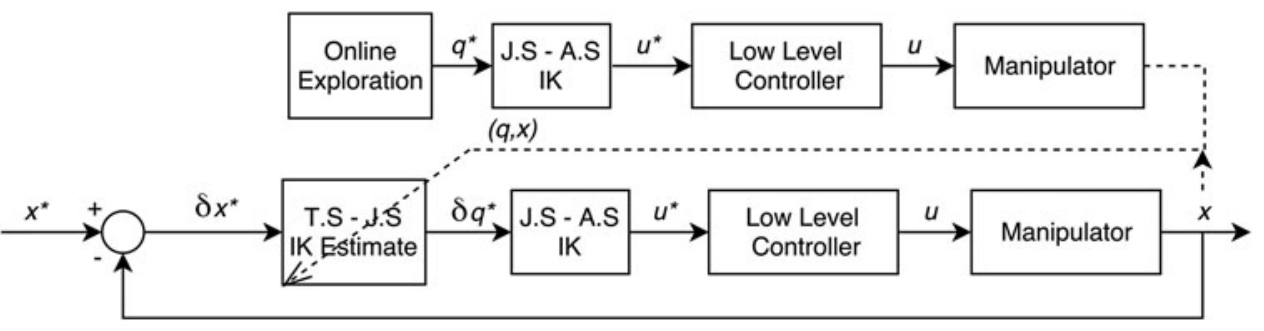

combining both model-based and model-free approaches was proposed in Refs. ${ }^{52-54}$ In Ref., ${ }^{52}$ the manipulator is modeled as multiple sections with one translational and two rotational DoF. Then, multiple neural networks are used to resolve redundancy and to obtain the mapping from the task space to the high-dimension configuration space. The configuration space to actuator space mapping is done analytically as it was found to be more straightforward. A noticeable limitation of such a method is the high sensory information required, which in the article, the authors have synthesized from certain empirical data.

A polar method was adopted in Ref., ${ }^{53}$ with the configuration space to task space mapping being analytically modeled using the PCC approximation. The actuator space to configuration space mapping is learned also considering possible first-order viscoelastic effects. A feedback strategy such as in Ref. ${ }^{41}$ was also used to provide high tracking accuracy, however, only for a planar manipulator. In Ref., ${ }^{54}$ it was shown that by learning only the model error incurred by an analytical model (a CC model), better forward and IK models could be obtained. In this way, it is also possible to leverage the advantages of an analytical model (such as null space motions) along with the generality of learning methods.

\section{Summary of model-free static controllers}

One of the primary advantages of model-free approaches is to circumvent the need to define the parameters of the configuration space and/or joint space and is independent of the manipulator shape. Due to this, arbitrarily complex kinematic models can be developed depending on the abundance of the sample data and sensory noise. This is probably why modelfree approaches have fared better for systems that are highly nonlinear, nonuniform, ${ }^{39}$ influenced by gravity, ${ }^{41,48}$ or act within unstructured environments where modeling is almost impossible. ${ }^{42}$ However, for well-behaved compact manipulators in known environments, model-based controllers are still more accurate and reliable. Furthermore, due to their black box nature, stability analysis and convergence proofs are difficult to establish. Static/kinematic controllers assume little or no dynamic coupling between sections.

As mentioned in the beginning, static/kinematic controllers rely on the steady-state assumption, which hinders accurate and fast motion of soft manipulators. Hence, controllers that consider the dynamic behavior of these manipulators are important for faster, dexterous, efficient, smoother tracking and in situations where coupling effects cannot be ignored.

\section{Model-Based Dynamic Controllers}

Probably the most challenging field in the control of continuum/soft robots is the development of nonstatic controllers that considers the complete dynamics of the whole manipulator. Development of dynamic controllers would require the formulation of the kinematic model and an associated dynamic formulation. The fact that kinematic models are difficult to develop themselves, a dynamic formulation based on these imprecise models, aggravates the model uncertainties. ${ }^{55}$ On the contrary, even if exact kinematic and dynamic models are available, an appropriate controller would then require high-dimensional sensory feedback. ${ }^{56}$ Moreover, some dynamic properties/disturbances are inherently uncontrollable due to their underactuated nature. ${ }^{14}$ Development of reliable parameter estimation algorithms and accurate sensory information is also crucial.

One of the first theoretical studies on the dynamic control of continuum robots was done in Ref. ${ }^{57}$ In Ref., ${ }^{57}$ it was validated through simulations of a planar single multisection continuum robot that a simple feedforward and feedback proportional derivative (PD) controller can achieve exponential tracking of a set point. The feedforward component inputs the actuator torques, satisfying the static holding torques, and the feedback component ensures the convergence of the set point position. A similar experimental study showed that a simple proportional controller can regulate the orientation of a planar continuum robot and a PD controller with coupling compensation can damp out manipulator vibrations. ${ }^{14}$ Nonetheless, these studies were conducted on simplified models that do not capture the true nonlinearities of continuum/soft robots.

The first closed-loop task space dynamic controller for continuum robots was demonstrated in Ref., ${ }^{58}$ although only by simulations. The kinematic for the $2 \mathrm{D}$ multisection robot was formulated using the $\mathrm{CC}$ model and the corresponding dynamic model in the configuration was presented in the Euler-Lagrangian form using lumped dynamic parameters. One main difference of such a model from the dynamic model of a rigid robot is the addition of the potential energy due to bending and extension (dependent only on the kinematic configuration). In this dynamic equation, the task space state variables can be substituted in place of the configuration state variables using the kinematic model. Note that by this way small errors in the kinematic model will exponentially rise when computing the higher order states and thereby affecting the accuracy of dynamic model. The implemented controller can be described as a PD-computed torque controller where the auxiliary control signal is represented in terms of the task space variables. An additional term for controlling the configuration space in the null space is also added. Although the robustness of the controller is shown by adding Gaussian white noise, the performance of such a controller can only be validated experimentally since it hinges on the CC approximation. However, the validity of the 
CC model for the same model was concurrently questioned in Ref. ${ }^{55}$ Furthermore, it must be brought to the attention of the reader that the stability proof was derived assuming that the kinematic and dynamic model is perfect.

A different control approach for the same kinematic and dynamic model, in simulation, was done using a sliding mode controller in Ref., ${ }^{59}$ however, only for closed-loop configuration space control. A first-order (assuming that the input/output relative degree is two) sliding surface is defined as the filtered tracking error for this purpose. The advantage of a sliding mode controller over a simple inverse dynamics-based PD controller is the higher robustness to model uncertainties; the downside being the slower error convergence, chattering, and higher gain requirements. An experimental evaluation of this method was conducted with a planar three-section continuum arm in Ref., ${ }^{60}$ along with comparisons to a simple feedback linearization-based PD controller in the configuration space. It was observed that the sliding mode controller performed better in terms of accuracy and speed indicating that model uncertainties were significant. In addition, a task space controller for teleoperation was demonstrated using the controller mentioned in Ref., ${ }^{58}$ which showed good tracking performance for a low-frequency reference.

Considering the fact that the actuator dynamics of pneumatic actuators is slower and more nonlinear than tendon-driven actuators, works focusing on optimal dynamic controllers for pneumatically actuated manipulators started to emerge. One such approach for trajectory optimization was demonstrated using simulations in Ref., ${ }^{61}$ where the objective was to estimate the optimal trajectory that reduces the transition time and actuator jerk. The nonlinear optimization problem is formulated with kinematic constraints (CC model), actuator dynamic constraints, and boundary constraints with the mass flow as the trajectory variable. Along the same lines, a trajectory optimization scheme for a comprehensive dynamic model of a soft planar manipulator was described in Ref. $^{62}$ (Fig. 10). Using the CC model for expressing the kinematics of the manipulator, a dynamic model was derived in the configuration space. A detailed derivation for calculating the generalized torques from the cylinder displacement and reference input is described in the article. A direct collocation approach is used to simultaneously identify the optimal generalized torques and corresponding manipulator state with the systems kinematics, dynamics, boundary conditions, and tracking objective as constraints. The objective function is to reduce the final end effector velocity. An optimization prob- lem is used for obtaining the optimal reference inputs to the actuator to realize the initial trajectory. Another advantage of a solving the control problem as an optimization problem is that it alleviates the need for a high-level path planner. The openloop policy was successfully able to reach statically unreachable target points with high probability; the first demonstration in the field of continuum/soft manipulators. Even then, an iterative learning control scheme to reidentify the system parameters was required in between trials for best performance.

Another comprehensive model-based controller, seemingly a variation of Ref., ${ }^{58}$ based on the dynamics of the joint space was proposed in Ref. ${ }^{63}$ The kinematics is based on the $\mathrm{CC}$ model and the dynamic model is represented in the joint space. A PD-computed torque controller in the joint space is proposed. To transform the generalized torques used in the dynamic model to the desired actuator pressures, an inversion scheme is proposed. Experimental results even without the PD term showed decent results, validating the dynamic model. An extension of Ref., ${ }^{63}$ which also considers the dynamics of the pneumatic chambers, was proposed in Ref. ${ }^{64}$ (Fig. 11). With this, an inner loop-decoupled PD-computed torque controller is cascaded to the existing controller. Consideration of the pneumatic dynamics is important because its response is slower and more nonlinear compared with the dynamics of electromagnetic actuators. Since the controller does not consider the actuator and kinematic constraints, the performance is currently limited.

A recent interesting approach in the field of soft robotic manipulators in terms of design and control was stated in Ref. ${ }^{65}$ This soft humanoid robot was constructed such that the joints are similar to traditional rotational joints. Therefore, the kinematics of the manipulator can be modeled like traditional rigid robots allowing for much simpler dynamics models, which are identified empirically. The authors have ignored gravitational and cross coupling effects, and the relationship between joint torques and pressure is derived. Due to the simplified model and design, a model predictive controller (MPC) in the joint space could be implemented at high frequency $(300 \mathrm{~Hz})$.

\section{Summary of model-based dynamic controllers}

Dynamic controllers are important for industrial applications where time and cost are also important along with the accuracy. Model-based dynamic controllers for continuum/soft manipulators are still in their nascent stage,

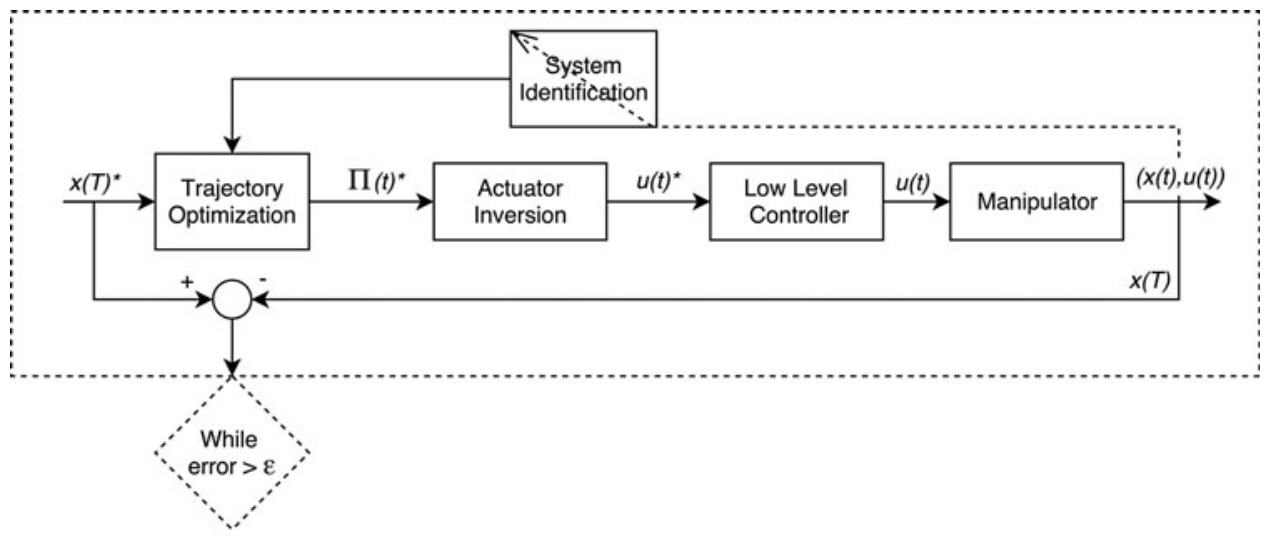

FIG. 10. Trajectory optimization algorithm for openloop dynamic task space control. 


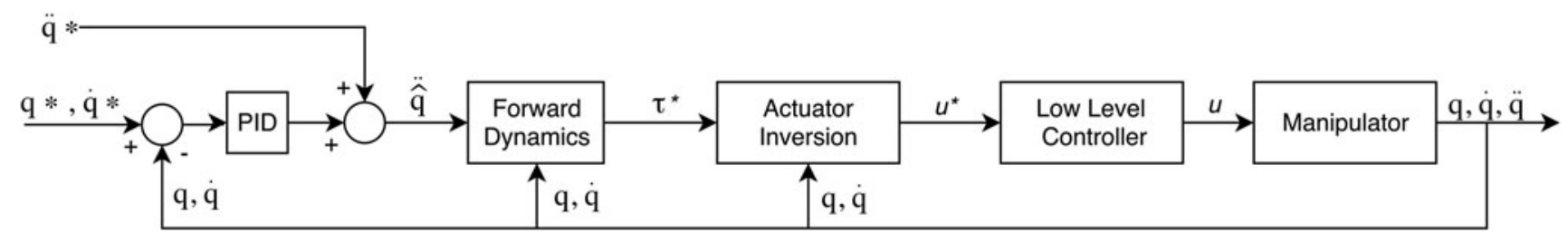

FIG. 11. Joint space dynamic controller by feedback linearization.

and consequently, there are a multitude of gaps that should be addressed in design, modeling, and control. Dynamic models directly mapping the control inputs (voltage, pressure, or encoder values) to the task space variables should provide the ideal performance for any model-based control approach. Currently, most of the dynamic control approaches are focused on the joint space control with an exception of few. ${ }^{62}$ Even in this case, due to the computational complexity, the controller had to be designed in open loop for a planar uniform manipulator. However, if the feedforward controller is perfect, this would be the most ideal choice. MPCs are ideal candidates for control of these continuum/soft manipulators, allowing for low-gain accurate control. Their application is currently limited only because of the computational complexity of the current dynamic models.

With the increase in computational power, sensing capabilities, and intelligent controllers, we can expect better developments in model-based dynamic controllers. Alternatively, another route to consider are machine learning-based approaches for learning open-loop controllers, for dynamic compensation, or for learning black box dynamic models.

\section{Model-Free Dynamic Controllers}

Model-free approaches for dynamic control of continuum/ soft manipulators are still a relatively unexplored area. Nonetheless, the earliest usage of machine learning techniques for control of continuum robots was implemented for compensating for dynamic uncertainties in Ref. ${ }^{66}$ (Fig. 12). However, the methodology was described only for closedloop dynamic control of the joint variables. The control architecture is composed of a feedback component, which is based on a continuous asymptotic tracking control strategy for uncertain nonlinear systems (similar to a second-order sliding mode controller), ${ }^{67}$ and a feedforward component made using neural networks. The objective of the neural network is to compensate for the dynamic uncertainties and thereby reducing the uncertainty bound that improves the performance of the feedback controller.
In the domain of reinforcement learning, a simulated multisegmented dynamical planar model of the octopus arm was developed in Ref. ${ }^{68}$ The authors then addressed the task of reaching a point by modeling the problem as a Hidden Markov Model that was solved online through a nonparametric Gaussian temporal difference learning algorithm. The underlying idea is to learn an action-value function via Bayesian inference from which an optimal control policy can be derived. In Ref., ${ }^{69}$ it was demonstrated that an actorcritic-based reinforcement learning approach could solve the same problem in the context of continuous action spaces. A significant challenge that, however, remains, to adopt such methods in practice, is to reduce the real-time costs for generating solutions.

Recently, the first direct actuator space to task space dynamic controller was experimentally demonstrated on a $3 \mathrm{D}$ soft pneumatic manipulator. ${ }^{70}$ The approach involved learning the forward dynamic model using a class of recurrent neural network and using trajectory optimization on the learned model. ${ }^{62}$ Such types of controllers reveal a different region of dynamic behavior that a soft manipulator can attain in terms of speed, workspace volume, and efficiency. The advantages of a model-free approach are clearly evident in terms of the ease of modeling accuracy and low sensory requirements. However, the controller is purely open loop due to the computational complexity and it was experimentally validated only on a single-section manipulator.

\section{Summary of model-free dynamic controllers}

To sum up, although model-free approaches offer a relatively simpler path for developing dynamic controllers, practical applications are limited either due to training time or stability concerns. ${ }^{71}$ Nonetheless, it is a possibility that should be looked upon, especially with the growth of more robust algorithms for training recurrent dynamic network. ${ }^{72}$ That being said, hybrid controllers that merge model-based and model-free approaches could also be a viable approach to consider.
FIG. 12. Model-free dynamic controller in the joint space.

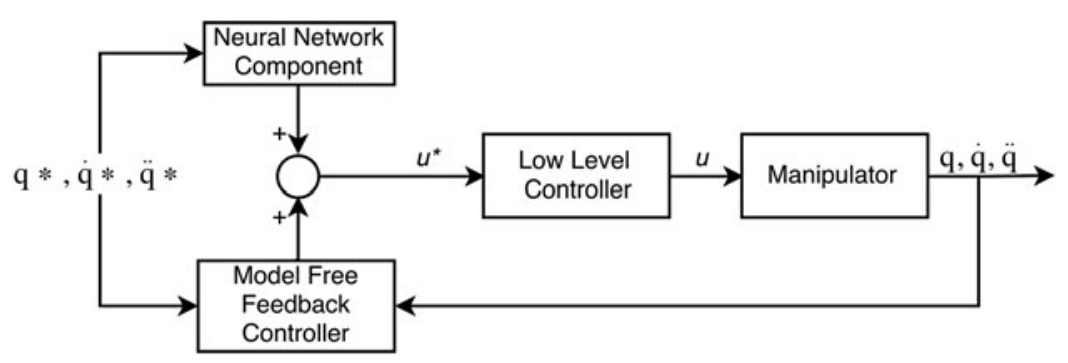




\section{Discussions}

From our survey on the current control approaches in soft robotics, it is apparent that the design of controllers for continuum/soft manipulators is not only application dependent but also influenced by the manipulator design, actuator and sensor availability. Therefore, it is difficult to compare and contrast all the approaches under the same umbrella. However, depending on the design, actuation, and application, there are some trends observed. Classification of a manipulator as continuum or soft did not affect the controller design; at least, it is not evident. This means that controllers developed for continuum manipulators can be easily transferred to their soft subgroup. Medical applications that rely on compact manipulators, manufactured with high precision, tend to use model-based approaches because of the reliability and highly controlled environment. Likewise, manipulators with nonuniform geometry and high nonlinearity tend to use model-free methods for a lack of better analytic models. For manipulation in unstructured environments, currently only model-free methods have shown promising results.

Another interesting observation is the absence of dynamic controllers developed for tendon-driven manipulators. This could be because of the nonuniform loading for cable actuation contrasting to the high damping and low force actuation provided by pneumatic actuators. Nonuniform loading occurs due to the physical interactions between the cable guide and the cable due to friction and this leads to irregular actuation of the manipulator DoFs. High damping coupled with low force actuation reduces overall energy supplied to the system therefore reducing the chaotic nature of the manipulator dynamics.

The controller regime to some extent depends on the sensor availability. For instance, closed-loop configuration space controllers require vision sensors. Model-based closedloop kinematic controllers for pneumatically actuated manipulators used wire cable potentiometers. This is because of joint space estimation for pneumatic actuation in not so straightforward-like rigid robots.

With regard to unexplored fields of research, clear voids are evident in hybrid control approaches and model-free approaches for dynamic control. Application of machine learning for learning the dynamic mapping from the actuator space to task space/configuration space is a viable method to be investigated. Similarly, hybrid learning approaches incorporating both model-based and model-free methods is a highly promising line of research. In addition, machine learning algorithms incorporating prior knowledge of the system would also provide a way for faster and more stable learning. ${ }^{73}$ Another overlooked topic is the importance of the low-level controllers (actuator dynamics) in the overall stability and response of the higher level control architecture.

Continuum/soft manipulators offer a technological solution to complex tasks in sensitive environments. Leveraged by their light weight, compact, and inherently safe structure, they can be used in various complex scenarios with elementary control strategies. ${ }^{9,10}$ Current trends in soft robot are individual efforts based on novel actuation, design, sensing, and control technologies for particular applications. However, an overlooked aspect is the interdependencies of these elements among themselves and with the environment. ${ }^{7}$ The possibility of outsourcing computational burden to the body (morphological computation) has been widely deliberated and even experimentally proven ${ }^{74}$ along with the effect of sensory feedback. ${ }^{75}$ In a control perspective, this corresponds to a zero lag adaptive feedback controller. Exploitation of this intrinsic controller has been achieved in some cases. ${ }^{5} \mathrm{We}$ believe that the future evolution of controllers for soft robotic manipulators would also be in this direction, where the morphological properties of the complex manipulators would also be utilized for more accurate, robust, and dexterous manipulation.

\section{Acknowledgments}

The authors would like to acknowledge the support by the People Programme (Marie Curie Actions) of the European Union's Seventh Framework Programme FP7/2007-2013/ under REA grant agreement Smart-e, number 608022 and the European Commission through the I-SUPPORT project (HORIZON 2020 PHC-19, \#643666.

\section{Author Disclosure Statement}

No competing financial interests exist.

\section{References}

1. Robinson G, Davies J. Continuum robots-a state of the art. Proceedings of the 1999 IEEE International Conference on Robotics and Automation (Cat. No. 99CH36288C), Detroit, MI, 1999.

2. Albu-Schaffer A, Eiberger O, Grebenstein M, Haddadin S, Ott C, Wimbock T, et al. Soft robotics. IEEE Robot Autom Mag 2008;3:20-30.

3. Laschi C, Mazzolai B, Cianchetti M. Soft robotics: technologies and systems pushing the boundaries of robot abilities. Sci Robot 2016;1:eaah3690.

4. Trivedi D, Rahn C, Kier W, Walker I. Soft robotics: biological inspiration, state of the art, and future research. Appl Bionics Biomech 2008;5:99-117.

5. Amend J, Brown E, Rodenberg N, Jaeger H, Lipson H. A positive pressure universal gripper based on the jamming of granular material. IEEE Trans Robot 2012;28:341-350.

6. Embodied Artificial Intelligence (Lecture Notes in Computer Science series, 3139) Iida F, Pfeifer R, Steels L, Kuniyoshi Y. (Eds). Berlin, Heidelberg; Springer: 2004.

7. Pfeifer R, Gómez G. Morphological computationconnecting brain, body, and environment. In: Creating BrainLike Intelligence. Lecture Notes in Computer Science, vol 5436. Sendhoff B, Körner E, Sporns O, Ritter H, Doya K. (Eds). Berlin, Heidelberg; Springer: 2009, pp. 66-83.

8. Manti M, Cacucciolo V, Cianchetti M. Stiffening in soft robotics: a review of the state of the art. IEEE Robot Autom Mag 2016;23:93-106.

9. Kim S, Laschi C, Trimmer B. Soft robotics: a bioinspired evolution in robotics. Trends Biotechnol 2013;31:287-294.

10. Rus D, Tolley M. Design, fabrication and control of soft robots. Nature 2015;521:467-475.

11. Walker I. Continuous backbone "continuum" robot manipulators. ISRN Robot 2013;2013:1-19.

12. Webster R, Jones B. Design and kinematic modeling of constant curvature continuum robots: a review. Int J Robot Res 2010;29:1661-1683.

13. Hannan M, Walker I. Kinematics and the implementation of an Elephant's Trunk Manipulator and other continuum style robots. J Robotic Syst 2003;20:45-63. 
14. Gravagne I, Rahn C, Walker I. Large deflection dynamics and control for planar continuum robots. IEEE/ASME Trans Mechatron 2003;8:299-307.

15. Gravagne I, Walker I. Manipulability, force, and compliance analysis for planar continuum manipulators. IEEE Trans Robot Autom 2002;18:263-273.

16. Jones B, Walker I. Kinematics for multisection continuum robots. IEEE Trans Robot 2006;22:43-55.

17. Trivedi D, Lotfi A, Rahn C. Geometrically exact models for soft robotic manipulators. IEEE Trans Robot 2008;24: 773-780

18. Bailly Y, Amirat Y. Modeling and control of a hybrid continuum active catheter for aortic aneurysm treatment. Proceedings of the 2005 IEEE International Conference on Robotics and Automation, Barcelona, Spain, 2005.

19. Camarillo D, Carlson C, Salisbury J. Configuration tracking for continuum manipulators with coupled tendon drive. IEEE Trans Robot 2009;25:798-808.

20. Camarillo DB, Carlson CR, Salisbury JK. Task-space control of continuum manipulators with coupled tendon drive. In: Experimental Robotics. Springer Tracts in Advanced Robotics, vol 54. Khatib O, Kumar V, Pappas GJ (Eds). Berlin, Heidelberg; Springer: 2009, pp. 271-280.

21. Xu K, Simaan N. Actuation compensation for flexible surgical snake-like robots with redundant remote actuation. Proceedings of the 2006 IEEE International Conference on Robotics and Automation (ICRA 2006), Orlando, FL, 2006.

22. Jones B, Walker I. Practical kinematics for real-time implementation of continuum robots. IEEE Trans Robot 2006; 22:1087-1099.

23. Bajo A, Goldman R, Simaan N. Configuration and joint feedback for enhanced performance of multi-segment continuum robots. 2011 IEEE International Conference on Robotics and Automation (ICRA), Shanghai, China, 2011.

24. Penning R, Jung J, Ferrier N, Zinn M. An evaluation of closedloop control options for continuum manipulators. 2012 IEEE International Conference on Robotics and Automation (ICRA), Saint Paul, MN, 2012.

25. Mahvash M, Dupont P. Stiffness control of surgical continuum manipulators. IEEE Trans Robot 2011;27:334-345.

26. Goldman R, Bajo A, Simaan N. Compliant motion control for continuum robots with intrinsic actuation sensing. 2011 IEEE International Conference on Robotics and Automation (ICRA), Shanghai, China, 2011.

27. Bajo A, Simaan N. Hybrid motion/force control of multibackbone continuum robots. Int J Robot Res 2015;35: 422-434.

28. Wang H, Chen W, Yu X, Deng T, Wang X, Pfeifer R. Visual servo control of cable-driven soft robotic manipulator. 2013 IEEE/RSJ International Conference on Intelligent Robots and Systems (IROS), Tokyo, Japan, 2013.

29. Mahl T, Hildebrandt A, Sawodny O. A variable curvature continuum kinematics for kinematic control of the bionic handling assistant. IEEE Trans Robot 2014;30:935-949.

30. Mahl T, Mayer A, Hildebrandt A, Sawodny O. A variable curvature modeling approach for kinematic control of continuum manipulators. 2013 American Control Conference, Washington, DC, 2013.

31. Till J, Bryson CE, Chung S, Orekhov A, Rucker DC. Efficient computation of multiple coupled Cosserat rod models for real-time simulation and control of parallel continuum manipulators. 2015 IEEE International Conference on Robotics and Automation (ICRA), Seattle, WA, 2015, pp. 5067-5074.
32. Boushaki M, Liu C, Poignet P. Task-space position control of concentric-tube robot with inaccurate kinematics using approximate Jacobian. 2014 IEEE International Conference on Robotics and Automation (ICRA), Hong Kong, China, 2014.

33. Largilliere F, Verona V, Coevoet E, Sanz-Lopez M, Dequidt J, Duriez C. Real-time control of soft-robots using asynchronous finite element modeling. 2015 IEEE International Conference on Robotics and Automation (ICRA), Seattle, WA, 2015.

34. Conrad B, Zinn M. Closed loop task space control of an interleaved continuum-rigid manipulator. 2015 IEEE International Conference on Robotics and Automation (ICRA), Seattle, WA, 2015.

35. Marchese A, Rus D. Design, kinematics, and control of a soft spatial fluidic elastomer manipulator. Int J Robot Res 2015;35:840-869.

36. Sadati SMH, Naghibi SE, Shiva A, Walker ID, Althoefer K, Nanayakkara T. Mechanics of continuum manipulators, a comparative study of five methods with experiments. In: Towards Autonomous Robotic Systems. TAROS 2017. Lecture Notes in Computer Science, vol 10454. Gao Y, Fallah S, Jin Y, Lekakou C (Eds). Cham, Switzerland; Springer: 2017, pp. 689-702.

37. Nguyen-Tuong D, Peters J. Model learning for robot control: a survey. Cogn Process 2011;12:319-340.

38. Giorelli M, Renda F, Ferri G, Laschi C. A feed-forward neural network learning the inverse kinetics of a soft cabledriven manipulator moving in three-dimensional space. 2013 IEEE/RSJ International Conference on Intelligent Robots and Systems (IROS), Tokyo, Japan, 2013.

39. Giorelli M, Renda F, Calisti M, Arienti A, Ferri G, Laschi C. Neural network and Jacobian method for solving the inverse statics of a cable-driven soft arm with nonconstant curvature. IEEE Trans Robot 2015;31:823-834.

40. Giorelli M, Renda F, Calisti M, Arienti A, Ferri G, Laschi C. Learning the inverse kinetics of an octopus-like manipulator in three-dimensional space. Bioinspir Biomim 2015; 10:035006.

41. Rolf M, Steil JJ. Efficient exploratory learning of inverse kinematics on a bionic elephant trunk. IEEE Trans Neural Netw Learn Syst 2014;25:1147-1160.

42. Yip M, Camarillo D. Model-less feedback control of continuum manipulators in constrained environments. IEEE Trans Robot 2014;30:880-889.

43. Yip M, Camarillo D. Model-less hybrid position/force control: a minimalist approach for continuum manipulators in unknown, constrained environments. IEEE Robot Autom Lett 2016;1:844-851.

44. Melingui A, Merzouki R, Mbede J, Escande C, Daachi B, Benoudjit N. Qualitative approach for inverse kinematic modeling of a Compact Bionic Handling Assistant trunk. 2014 International Joint Conference on Neural Networks (IJCNN), Beijing, China, 2014.

45. Melingui A, Lakhal O, Daachi B, Mbede J, Merzouki R. Adaptive neural network control of a compact bionic handling arm. IEEE/ASME Trans Mechatron 2015;20:2862-2875.

46. Thuruthel T, Falotico E, Cianchetti M, Laschi C. Learning global inverse kinematics solutions for a continuum robot. In: Parenti-Castelli V, Schiehlen W. (Eds). ROMANSY 21Robot Design, Dynamics and Control. Cham, Switzerland: Springer, 2016, pp. 47-54.

47. Thuruthel T, Falotico E, Cianchetti M, Renda F, Laschi C. Learning global inverse statics solution for a redundant soft 
robot. Proceedings of the 13th International Conference on Informatics in Control, Automation and Robotics, Lisbon, Portugal, 2016.

48. George Thuruthel T, Falotico E, Manti M, Pratesi A, Cianchetti M, Laschi C. Learning closed loop kinematic controllers for continuum manipulators in unstructured environments. Soft Robot 2017;4:285-296.

49. Malekzadeh M, Calinon S, Bruno D, Caldwell D. Learning by imitation with the STIFF-FLOP surgical robot: a biomimetic approach inspired by octopus movements. Robot Biomim 2014;1:13.

50. Ansari Y, Manti M, Falotico E, Cianchetti M, Laschi C. Multiobjective optimization for stiffness and position control in a soft robot arm module. IEEE Robot Autom Lett 2017;3:108-115.

51. Qi P, Liu C, Ataka A, Lam H, Althoefer K. Kinematic control of continuum manipulators using a fuzzy-model-based approach. IEEE Trans Industr Electron 2016;63:5022-5035.

52. Lakhal O, Melingui A, Merzouki R. Hybrid approach for modeling and solving of kinematics of a compact bionic handling assistant manipulator. IEEE/ASME Trans Mechatron 2016;21:1326-1335.

53. Jiang H, Wang Z, Liu X, Chen X, Jin Y, You X, et al. A two-level approach for solving the inverse kinematics of an extensible soft arm considering viscoelastic behavior. 2017 IEEE International Conference on Robotics and Automation (ICRA), Singapore, Singapore, 2017, pp. 6127-6133.

54. Reinhart R, Shareef Z, Steil J. Hybrid analytical and datadriven modeling for feed-forward robot control. Sensors (Basel) 2017;17:E311.

55. Trivedi D, Lotfi A, Rahn C. Geometrically exact dynamic models for soft robotic manipulators. 2007 IEEE/RSJ International Conference on Intelligent Robots and Systems (IROS), San Diego, CA, 2007.

56. Renda F, Giorelli M, Calisti M, Cianchetti M, Laschi C. Dynamic model of a multibending soft robot arm driven by cables. IEEE Trans Robot 2014;30:1109-1122.

57. Gravagne I, Walker I. Uniform regulation of a multisection continuum manipulator. Proceedings of the 2002 IEEE International Conference on Robotics and Automation (Cat. No. 02CH37292), Washington, DC, 2002.

58. Kapadia A, Walker I. Task-space control of extensible continuum manipulators. 2011 IEEE/RSJ International Conference on Intelligent Robots and Systems (IROS), San Francisco, CA, 2011.

59. Kapadia A, Walker I, Dawson D, Tatlicioglu E. A modelbased sliding mode controller for extensible continuum robots. Proceedings of the 9th WSEAS International Conference on Signal Processing, Robotics and Automation (ISPRA'10), Cambridge, UK, 2010, pp. 113-120.

60. Kapadia A, Fry K, Walker I. Empirical investigation of closed-loop control of extensible continuum manipulators. 2014 IEEE/RSJ International Conference on Intelligent Robots and Systems, Chicago, IL, 2014.

61. Falkenhahn V, Hildebrandt A, Sawodny O. Trajectory optimization of pneumatically actuated, redundant continuum manipulators. 2014 American Control Conference (ACC), Portland, OR, 2014.

62. Marchese A, Tedrake R, Rus D. Dynamics and trajectory optimization for a soft spatial fluidic elastomer manipulator. Int J Robot Res 2015;35:1000-1019.
63. Falkenhahn V, Hildebrandt A, Neumann R, Sawodny O. Model-based feedforward position control of constant curvature continuum robots using feedback linearization. 2015 IEEE International Conference on Robotics and Automation (ICRA), Seattle, WA, 2015.

64. Falkenhahn V, Hildebrandt A, Neumann R, Sawodny O. Dynamic control of the bionic handling assistant. IEEE/ ASME Trans Mechatron 2016;22:6-17.

65. Best C, Gillespie M, Hyatt P, Rupert L, Sherrod V, Killpack M. A new soft robot control method: using model predictive control for a pneumatically actuated humanoid. IEEE Robot Autom Mag 2016;23:75-84.

66. Braganza D, Dawson D, Walker I, Nath N. A neural network controller for continuum robots. IEEE Trans Robot 2007;23: 1270-1277.

67. Xian B, Dawson D, DeQueiroz M, Chen J. A continuous asymptotic tracking control strategy for uncertain nonlinear systems. IEEE Trans Autom Control 2004;49: 1206-1206.

68. Engel Y, Szabo P, Volkinshtein D. Learning to control an octopus arm with Gaussian process temporal difference methods. Advances in Neural Information Processing Systems (NIPS), Vancouver, Canada, 2005.

69. Silver D, Lever G, Heess, N, Degris T, Wierstra D, Riedmiller M. Deterministic policy gradient algorithms. Proceedings of the 31st International Conference on Machine Learning, Beijing, China, 2014.

70. Thuruthel TG, Falotico E, Renda F, Laschi C. Learning dynamic models for open loop predictive control of soft robotic manipulators. Bioinspir Biomim 2017;12: 066003.

71. Venkatraman A, Herbert M, Bagnell J. Improving multi-step prediction of learned time series models. Twenty-Ninth AAAI Conference on Artificial Intelligence (AAAI), Austin, Texas, 2015.

72. Bengio S, Vinyals O, Jaitly N, Shazeer N. Scheduled sampling for sequence prediction with recurrent neural networks. NIPS, Montreal, Canada, 2015.

73. Nguyen-Tuong D, Peters J. Model learning for robot control: a survey. Cogn Process 2011;12:319-340.

74. Nakajima K, Hauser H, Kang R, Guglielmino E, Caldwell D, Pfeifer R. A soft body as a reservoir: case studies in a dynamic model of octopus-inspired soft robotic arm. Front Comput Neurosci 2013;7:91.

75. Hauser H, Ijspeert A, Füchslin R, Pfeifer R, Maass W. The role of feedback in morphological computation with compliant bodies. Biol Cybern 2012;106:595-613.

Address correspondence to:

Thomas George Thuruthel

The Biorobotics Institute

Scuola Superiore Sant'Anna

Polo Sant'Anna Valdera

Viale Rinaldo Piaggio, 34

Pisa 56025

Italy

E-mail: t.thuruthel@sssup.it 